\title{
A practical resource-searching method for manufacturing grid
}

\author{
Jingzhi Fu
}

Received: 12 March 2014 / Accepted: 18 May 2014 /Published online: 1 June 2014

(C) The Author(s) 2014. This article is published with open access at Springerlink.com

\begin{abstract}
Manufacturing grid has become more and more popular because it can realize the resource share and collaboration within the worldwide scope. In most cases, the resource and task information in manufacturing grid is fuzzy and uncertain, but the resource-searching methods now are focused on deterministic value and have some limitations for manufacturing grid. This paper proposes a unified information model in which the information parameters are described as a set of interval values, and the conversion rules from the given capability parameters into intervals are discussed. On these bases, a new optimized resource-searching method is designed to find the best required manufacturing resource for the task by calculating the interval distance of the task and the resources. The method is verified to be effective by an instance. Furthermore, it is practical by comparing it with other resource-searching methods.
\end{abstract}

Keywords Manufacturing grid $\cdot$ Resource-searching method $\cdot$ Conversion rules $\cdot$ Interval distance

\section{Introduction}

Networked manufacturing mode has changed from CIM and $\mathrm{CE}$ to intelligent manufacturing, agile manufacturing, and disperse networked manufacturing with the application of network and information technology since the 1970s. Correspondingly, manufacturing resources share from the interior of the enterprise to alliance. Recently, grid and cloud manufacturing began to develop to realize that manufacturing resources share and collaborate within the global scope [1].

J. Fu $(\bowtie)$

School of information and control, Nanjing University of Information Science \& Technology, Nanjing 210044, China e-mail: jennyfjz@163.com
How to search for the suitable resource efficiently from so many diverse resources to meet with the requirements is one of the important research fields of manufacturing grid. Many scholars have studied some resource scheduling and allocation methods and put forward the corresponding algorithms. Zhang et al. [2] studied an ontology-based approach of automated service chaining for manufacturing grid. Tan and Fan [3] researched semantic-based service match and composition under networked manufacturing environment. Tao et al. [4, 5] researched manufacturing grid resource service QoS modeling and proposed QoS-based resource search method. A TQCSbased method was proposed by Liu et al. [6] and Shi et al. [7]. Yu et al. [8] and Wen et al. [9] proposed a multi-agent-based manufacturing grid search method. Deng et al. [10] and Chen et al. [11] proposed a resource characteristic-based scheduling strategy and applied it into the development of customized artificial joint. Zhang et al. [12] proposed a multi-objective optimization mathematical model and designed a GA-based manufacturing grid resource scheduling process. Lv et al. [13] researched a marked equilibrium-based manufacturing grid resource optimal allocation method. There are now four types of resource-searching methods in manufacturing grid: exhaustive searching method, centralized searching method, routing transfer method, and hierarchy-searching method [14], but these methods studied the resource-searching problem in manufacturing grid environment using quantitative or qualitative method or their combination. The resource information and the evaluation indicators are described as determined values. In fact, the networked resource and task information are often uncertain, fuzzy, redundant, and deficient. The author of this article discovered by research that it is an available method to use interval values to describe the uncertain information. Since the 1960s, some scholars have made a lot of pioneering work in the field of interval mathematics. Eldon and William [15] discussed global optimization problem based on interval analysis, but it seems difficult to solve practical engineering problems due to its 
complexity. In this paper, a new resource-searching method based on interval uncertain parameters will be discussed to search for the best resource in manufacturing grid.

The rest of this paper is organized as follows: In Section 2, the information of the manufacturing resources and tasks is modeled. In Section 3, some rules of conversion from uncertain parameters of manufacturing resource to interval values are proposed. The interval optimized searching method based on uncertain parameters will be discussed in detail in Section 4. At last, in Section 5, the method is testified effective and practical by contrast with the other resource-searching methods.

\section{Definitions}

Definition 1: manufacturing resource model In manufacturing grid, manufacturing resource model can be expressed as a two-dimensional tuple, i.e.:

$\mathrm{MR}=\{\mathrm{MRG}, \mathrm{MRA}\}$

where MRG is the set of the basic information including the name, function, and the number of the manufacturing resource. MRA is a collection of the capability information of the manufacturing resource describing the cost of usage, manufacturing cycle, quality of service, and service attributes of the resource.

Definition 2: manufacturing task demand model In manufacturing grid, the information that the manufacturing task demand on the resources can also be expressed as a twodimensional tuple, i.e.:

$\mathrm{MT}=\{\mathrm{MTG}, \mathrm{MTA}\}$

MTG is a collection of the basic information that the manufacturing task requires, including the task's name, function, and quantity that the task demands for the resources. MTA is a collection of the manufacturing task constraint on the resource's capability information, which describes the manufacturing cost, cycle, quality of service, and service attributes that the task demands.

Definition 3: manufacturing resource capability parameter domain It is the range that the manufacturing resource attribute parameter may reach according to the request of a type of task in manufacturing grid. Usually, each parameter's range can be represented or transformed into the form of interval as:

$$
\begin{aligned}
\mathrm{MRA}_{i} & =\left\{R_{i 1}, R_{i 2}, \ldots, R_{i m}\right\} \\
& =\left\{\left[\text { Lower Bound } R_{i j}, \text { Upper Bound } R_{i j}\right\}\right. \\
& =\left\{\left[\mathrm{LBR}_{i j}, \mathrm{UBR}_{i j}\right]\right\} j=1,2, \ldots, m
\end{aligned}
$$

Definition 4: task demand capability parameter domain In manufacturing grid, the capability parameter is required within a value range when the customer put forward a request to a suitable manufacturing resource for a task. Usually, each parameter range can be expressed as the form of interval as:

$$
\begin{aligned}
\text { MTA } & =\left\{T_{1}, T_{2}, \ldots, T_{m}\right\} \\
& =\left\{\left[\text { Lower Bound } T_{i j}, \text { Upper Bound } T_{i j}\right]\right\} \\
& =\left\{\left[\mathrm{LBR}_{i j}, \mathrm{UBR}_{i j}\right]\right\} j=1,2, \ldots, m
\end{aligned}
$$

Definition 5: manufacturing resource complete capability parameter domain In manufacturing grid, the capability parameter value of the manufacturing resource can reach a certain range according to the specific request task. Usually, each parameter range can be expressed as the form of interval too.

$$
\begin{aligned}
R T_{i} & =\left\{R_{i 1}^{(t)}, R_{i 2}^{(t)}, \cdots, R_{i m}^{(t)}\right\} \\
& =\left\{\left[\mathrm{LRB}_{i j}^{(t)}, \mathrm{UBR}_{i j}^{(t)}\right]\right\} j=1,2, \ldots, m
\end{aligned}
$$

Theorem If a manufacturing resource MR can accomplish a request task MT, the manufacturing resource complete capability parameter domain must be a subset of both the capability parameter domain of the manufacturing resource and demand capability parameter domain of the request task. That is, each complete capability parameter interval of the manufacturing resource is the intersection of its corresponding capacity parameter interval and the request task demand capability parameter interval. The formalized description is:

$$
\begin{aligned}
\mathrm{RT}_{i} & =\mathrm{MRA}_{i} \cap \mathrm{MTA} \\
& =\left\{\left[\operatorname{Max}\left(\mathrm{LBR}_{i j}, \mathrm{LBT}_{j}\right), \operatorname{Min}\left(\mathrm{UBR}_{i j}, \mathrm{UBT}_{j}\right)\right]\right\} j=1,2, \ldots, m
\end{aligned}
$$

Corollary For a manufacturing resource and manufacturing task, if there is a capability parameter satisfying: $\operatorname{Max}\left(L B R_{i j}\right.$, $\left.L B T_{j}\right)>\operatorname{Min}\left(U B R_{i j}, U B T_{j}\right) j \in(1,2, \cdots, m)$

then the manufacturing resource $M R_{i}$ cannot be the candidate resource for the task MT.

\section{Conversion rules}

Because manufacturing resource information in many cases is fuzzy and uncertain, it needs to be transferred first into the interval value to simplify the problem. It is an assumption that each demand capability parameter domain to a 
manufacturing task already is an interval which is expressed as $T=\left\{\left[\right.\right.$ LowerBound $T_{j}$, UpperBound $\left.\left.T_{j}\right]\right\} j=1$, $2, \cdots, m$ The interval conversion rules of the manufacturing resource are as follows:

\subsection{Conversion of non-interval parameters}

The parameter may be a deterministic value, limited value, or unlimited value when it is non-interval:

Rule 1 If a capability parameter of the manufacturing resource is a deterministic value or estimated value, namely $R_{i j}=r_{i j}$, (or $R_{i j} \approx r_{i j}$ ), it should be transformed into an interval. The upper and lower bounds of the interval are the same and equal to the exact value. That is, $R_{i j}=\left[L B R_{i j}, U B R_{i j}\right]$ and $L B R_{i j}=U B R_{i j}=r_{i j}$.

Rule 2 If a capability parameter of the manufacturing resource is a limited value, namely $R_{i j} \geq($ or $>) r_{i j}$, then if $r_{i j}>$ UpperBound $T_{j}$, the manufacturing resource cannot be the candidate resource for the task of $T$ according to the corollary provided in Section 2. Otherwise, it should be transferred into $R_{i j}=\left[\mathrm{LBR}_{i j}, \mathrm{UBR}_{i j}\right]$, and $\mathrm{LBR}_{i j}=r_{i j}, \mathrm{UBR}_{i j}=\mathrm{UBT}_{j}$.

Rule 3 If a capability parameter of the manufacturing resource is a limited value, namely $R_{i j} \leq($ or $<) r_{i j}$, then if $r_{i j}<$ LowerBound $T_{j}$, the manufacturing resource cannot be the candidate resource for this task. Otherwise, it should be transferred into $R_{i j}=\left[\mathrm{LBR}_{i j}, \mathrm{UBR}_{i j}\right]$ and $\mathrm{LBR}_{i j}=\mathrm{LBT}_{j}, \mathrm{UBR}_{i j}=r_{i j}$.

Rule 4 If a capability parameter of the manufacturing resource is not limited, the parameter domain of the task should be instead of the resource parameter domain. That is, $R_{i j}=\left[\mathrm{LBR}_{i j}, \mathrm{UBR}_{i j}\right]$ and $\mathrm{LBR}_{i j}=$ $\mathrm{LBT}_{j}, \mathrm{UBR}_{i j}=\mathrm{UBT}_{j}$.

\subsection{Conversion of interval parameters}

When a parameter is interval, it will be a closed interval, open interval, or out of the interval that the task required, so the following conversion rules should be used:

Rule 5 If a parameter of the manufacturing resource is a closed interval, i.e., $R_{i j}=\left[L B R_{i j}, U B R_{i j}\right]$, no conversion is required. Otherwise, if a capability parameter is an open interval (one or both ends), the open interval is needed to be transferred into the corresponding closed interval.

Rule 6 If a parameter domain meets $R_{k j} \cap T_{j} j \in(1,2, \cdots, m)=\Phi$, then the resource $M R_{k}$ is not the candidate resource. Only if all the parameters' domain of a certain manufacturing resource satisfy that $R_{i j} \cap T_{j} \neq \Phi(j=1,2$, $\cdots, m)$, the resource $M R_{i}$ might be the candidate resource for the task and the following transformation should be performed:

$$
\begin{aligned}
R_{i j}= & {\left[\mathrm{LBR}_{i j} \mathrm{UBR}_{i j}\right]=R A_{i} \cap T A } \\
= & \left\{\left[\operatorname{Max}\left(\mathrm{LBR}_{i j}, \mathrm{LBT}_{j}\right), \operatorname{Min}\left(\mathrm{UBR}_{i j}, \mathrm{UBT}_{j}\right)\right]\right\} \\
& (j=1,2, \ldots, m)
\end{aligned}
$$

After the above transformation, it can be verified that there is the following relationship between arbitrary resource capability parameter and the task demand capability parameter:

$\mathrm{LBR}_{i j} \geq \mathrm{LBT}_{j}$ and $\mathrm{UBR}_{i j} \leq \mathrm{UBT}_{j}$

Now, we can transfer each parameter domain into the interval between the value of $[0,1]$.

Rule 7 Set $\left[\right.$ LowerBound $T_{j}$, UpperBound $\left.T_{j}\right]=[0,1]$, then

$$
R_{i j}=\left[\mathrm{LBR}_{i j}, \mathrm{UBR}_{i j}\right]=\left[\frac{\mathrm{LBR}_{i j}-\mathrm{LBT}_{j}}{\mathrm{UBT}_{j}-\mathrm{LBT}_{j}}, \frac{\mathrm{UBR}_{i j}-\mathrm{LBT}_{j}}{\mathrm{UBT}_{j}-\mathrm{LBT}_{j}}\right]
$$

\section{Resource-searching method}

\subsection{Mapping from manufacturing task to the resource}

In manufacturing grid, the process of mapping from manufacturing task to manufacturing resource is a process of matching the demand capability parameters of the manufacturing task to the capability parameters of the manufacturing resource. The best manufacturing resource is found to complete a specific task by considering the value range of each parameter. In general, the set of the candidate resources should be determined firstly. Then, the scope of searching is narrowed gradually because there are too many manufacturing resources in the networked environment. At last, according to a certain algorithm, to find the best resource from the candidate resources, this process can be expressed as:

$\left.\mathrm{MTA} \rightarrow\left\{\mathrm{MRA}_{c}\right\}_{(c=1,2, \ldots, q}\right) \rightarrow \ldots \rightarrow \mathrm{MRA}_{k}$

The resource $M R_{k}$ is the best manufacturing resource for the task MT. The $\left\{\mathrm{MRA}_{c}\right\}_{(c=1,2, \ldots, q)}$ are the $q$ candidate resources getting from the $n$ resources after the initial screening, where $q \leq n$.

\subsection{Resource-searching procedures}

There may be a lot of resources in manufacturing grid, but it will cost much time to search for the best ones using the exhaustive method. Therefore, the resources can be clustered first in order to make the similar resources belong to the same 
cluster unit, then find the cluster unit that has the nearest interval distance with the task as candidate resources, and finally search the nearest resource from the candidate resource as the best manufacturing resource. The specific procedures are shown in Fig. 1 and follows:

Step 1 To convert the resources and task capability parameters into values between 0 and 1 . That is, the parameters are transformed into interval values first, then normalized into $[0,1]$.

Step 2 To compute the similarity between every two manufacturing resources using:

$$
\left.d_{i j}=\frac{1}{m} \sum_{k=1}^{m}\left\{\operatorname{Max}\left(\left|\mathrm{LBR}_{i k}-\mathrm{LBR}_{j k}\right|,\left|\mathrm{UBR}_{i k}-\mathrm{UBR}_{j k}\right|\right)\right]\right\}
$$

Step 3 In reference to the method proposed in [14], different clustering results $A_{l}(l=1,2, \cdots, q)$ can be obtained according to the similarity matrix and a set of different intercept, where $q$ is the number of the cluster units.

Step 4 The clustering center for each cluster unit can be calculated using Eq. 4.2.

$$
\begin{aligned}
& A_{l}=\left\{\left[\mathrm{LBA}_{l k}, \mathrm{UBA}_{l k}\right]\right\}
\end{aligned}
$$

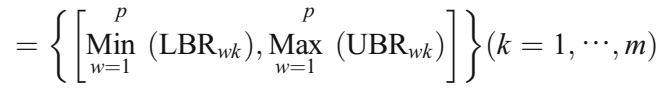

where $p$ is the number of the manufacturing resources in the cluster unit $A_{l}$.

Step 5 To calculate the closeness named interval distance between the cluster center of each cluster unit and the manufacturing task. The formula is:

$$
d_{A T}^{l}=\sum_{j=1}^{m}\left\{\lambda j \cdot\left[\delta 1 \cdot \mathrm{LBA}_{l j}+\delta 2 \cdot\left(1-\mathrm{UBA}_{l j}\right)\right]\right\}(l=1, \cdots, q)
$$

where $\lambda j$ is the weight of the number $j$ parameter. For the parameter of the smaller, the better $\delta 1=1, \delta 2=0$, while for the

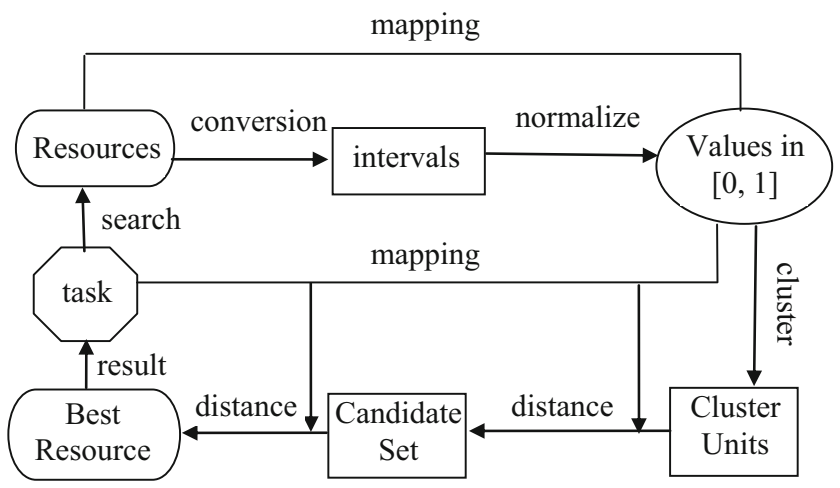

Fig. 1 The optimized searching procedures parameter of the larger, the better $\delta 1=0, \delta 2=1$. The best resource will be in the clustering unit of $A s$ with the smallest closeness value of $d_{A T}^{l}$, which is called the candidate resources set.

Step 6 To calculate the closeness of the manufacturing task to each candidate resource in $A s$. The formula is:

$$
d_{r t}^{s}=\sum_{j=1}^{m}\left\{\lambda j \cdot\left[\delta 1 \cdot \mathrm{LBR}_{s j}+\delta 2 \cdot\left(1-\mathrm{UBR}_{s j}\right)\right]\right\}(s=1, \cdots, p)
$$

The resource with the smallest closeness value of $d_{r t}^{s}$ is the best resource.

\subsection{Case study}

It is assumed that the capability information of a manufacturing task and the resources which can proceed the task in manufacturing grid system is described as shown in Table 1.

We can see from the table that the parameters are deterministic, estimated, default, or interval, so they should be converted into intervals and then normalized according to the rules proposed in Section 3. The normalized values shown in Table 2 eliminate the differences of the four types of the parameters so as to select the best resource taking them all into account.

According to the experience, "time" and "cost" parameters are "the smaller-the-better" type of indicators while "quality" and "service" are "the bigger-the-better" type of indicators, so the interval distance of the task to each resource can be calculated using Eq. 4.4.

Figure 2 is the comparison of the closeness when all the parameters are taken into account equally, which is shown as a curve, and only one parameter is taken into account

Table 1 Capability information of the manufacturing task and the resources

\begin{tabular}{llllll}
\hline Capability info & Time (h) & Quality & Cost (Yuan) & Service \\
\hline Resources & $R_{1}$ & $\leq 35$ & $\geq 0.86$ & 143 & {$[0.63,0.99]$} \\
& $R_{2}$ & About 21 & $\geq 0.52$ & $\leq 163$ & {$[0.51,0.77]$} \\
& $R_{3}$ & {$[20,27]$} & {$[0.85,0.9]$} & {$[120,170]$} & {$[0.72,0.79]$} \\
& $R_{4}$ & {$[20,25]$} & {$[0.6,0.9]$} & {$[128,158]$} & 0.86 \\
& $R_{5}$ & {$[26,35]$} & {$[0.55,0.9]$} & {$[126,133]$} & - \\
& $R_{6}$ & {$[25,33]$} & {$[0.71,0.8]$} & {$[103,125]$} & {$[0.8,0.93]$} \\
& $R_{7}$ & 28 & 0.95 & {$[90,120]$} & about 0.56 \\
& $R_{8}$ & {$[19,30]$} & - & $\geq 183$ & 0.68 \\
& $R_{9}$ & 20 & 0.67 & {$[143,155]$} & 0.93 \\
Task & $R_{10}$ & $\geq 30$ & {$[0.8,0.95]$} & {$[158,183]$} & {$[0.65,0.72]$} \\
\hline & $T$ & {$[16,36]$} & {$[0.5,1]$} & {$[93,193]$} & {$[0.3,1]$} \\
\hline
\end{tabular}


Table 2 the normalized parameter domain

\begin{tabular}{llllll}
\hline Capability info & Time & Quality & Cost & Service \\
\hline Resources & $R_{1}$ & {$[0,0.95]$} & {$[0.72,1]$} & {$[0.5,0.5]$} & {$[0.47,0.99]$} \\
& $R_{2}$ & {$[0.25,0.25]$} & {$[0.04,1]$} & {$[0,0.7]$} & {$[0.3,0.67]$} \\
& $R_{3}$ & {$[0.2,0.55]$} & {$[0.7,0.8]$} & {$[0.27,0.77]$} & {$[0.6,0.7]$} \\
& $R_{4}$ & {$[0.2,0.45]$} & {$[0.2,0.8]$} & {$[0.35,0.65]$} & {$[0.8,0.8]$} \\
& $R_{5}$ & {$[0.5,0.95]$} & {$[0.1,0.8]$} & {$[0.33,1]$} & {$[0,1]$} \\
& $R_{6}$ & {$[0.45,0.85]$} & {$[0.42,0.6]$} & {$[0.1,0.32]$} & {$[0.71,0.9]$} \\
& $R_{7}$ & {$[0.6,0.6]$} & {$[0.9,0.9]$} & {$[0,0.27]$} & {$[0.37,0.37]$} \\
& $R_{8}$ & {$[0.15,0.7]$} & {$[0,1]$} & {$[0.9,1]$} & {$[0.54,0.54]$} \\
& $R_{9}$ & {$[0.2,0.2]$} & {$[0.34,0.34]$} & {$[0.5,0.62]$} & {$[0.9,0.9]$} \\
& $R_{10}$ & {$[0.7,1]$} & {$[0.6,0.9]$} & {$[0.65,0.9]$} & {$[0.5,0.6]$} \\
& $T$ & {$[0,1]$} & {$[0,1]$} & {$[0,1]$} & {$[0,1]$} \\
\hline
\end{tabular}

respectively, which is expressed as points in the figure. We can see from Fig. 2 that the points has distributed in both sides of the curve. It illustrates that it is a compromise if all the parameters are taken into account. Certainly, the weight of each parameter the user required may be different. The searching result is different accordingly. Figure 3 is the resource-searching result with the three different sets of parameter weights. Trends of the three curves are the same and $\mathrm{R} 1$ is almost the best resource, so the resource-searching method proposed in this paper is effective.

\section{Complexity of the method}

From the procedures proposed in Section 4.2, we can see that the method in this paper is similar with the hierarchy-

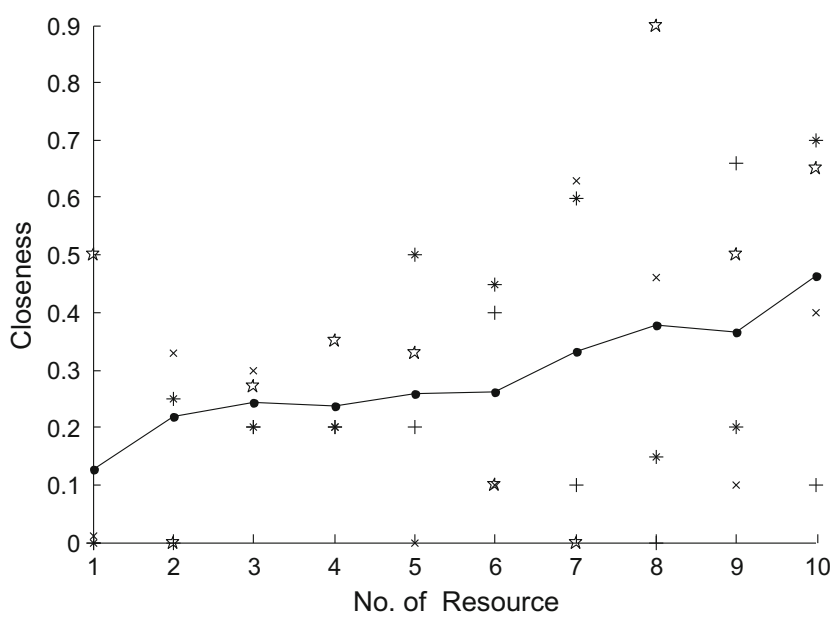

Fig. 2 The closeness of the task to each resource. Points with asterisks express only time that is taken into account. Points with multiplication signs express only quality that is taken into account. Points with stars express only cost that is taken into account. Points with plus sign express only service that is taken into account. The curve expresses that each parameter is taken into account equally

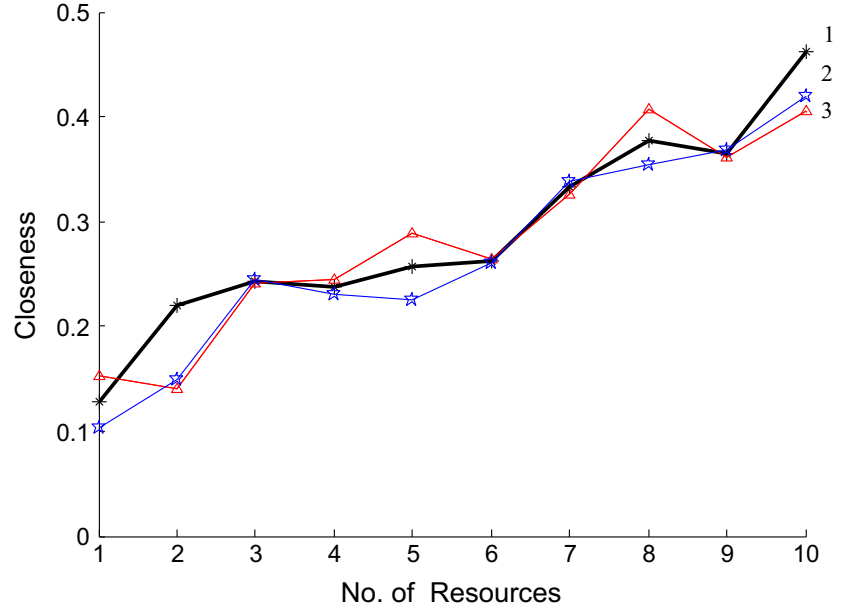

Fig. 3 The closeness of the task to each resource with different weight of parameters. Curve 1 is the closeness curve when $\lambda 1=\lambda 2=\lambda 3=\lambda 4=0.25$. Curve 2 is the closeness curve when $\lambda 1=\lambda 3=0.2, \lambda 2=\lambda 4=0.3$. Curve 3 is the closeness curve when $\lambda 1=\lambda 3=0.3, \lambda 2=\lambda 4=0.2$

searching method (HSM) except that it increases by a procedure of inversion, so its complexity is greater than HSM by W, which is the longest length from the request node to the resource nodes.

The comparison of the five resource-searching methods is shown in Fig. 4. We can see from Fig. 4 that the complexity of the method based on interval proposed in this paper is smaller than the other methods except for HSM when the number of the resources $n>$ $2.25 \mathrm{~W}$. On the other hand, the result of the interval method proposed in this paper is closer to the reality because the algorithm uses interval parameters instead of deterministic values, so it is more practical and suitable to resource searching in manufacturing grid where there are much more quantities and types of resources.

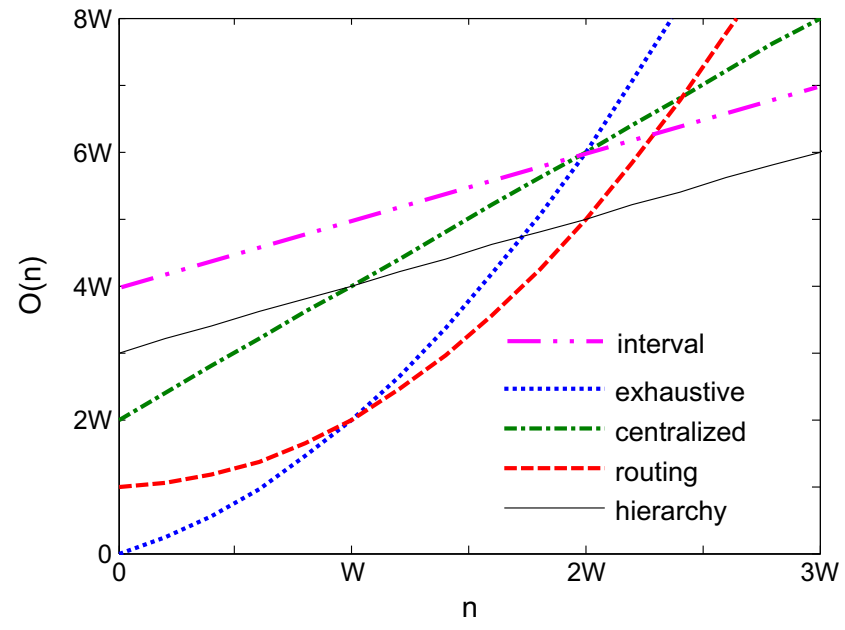

Fig. 4 The complexity of the five methods 


\section{Conclusion}

There are so many resources in manufacturing grid that the resource-searching method becomes more important and difficult. Based on interval analysis, the information model is established, and the transformation rules from the information parameters to intervals are discussed in detailed in this paper. On the basis of these, a new method is designed to find the best manufacturing resource for specific task by calculating the interval distance. It is verified that the method is effective and practical compared with the other resource-searching methods for a single manufacturing task, but tasks that require the same resource at the same time may be more than one because the network environment is complex and diverse. How to coordinate between these tasks and resources to achieve global optimization and how to solve the resource confliction problem will be the research content subsequently.

Acknowledgments This research is supported by National Natural Science Foundation of China (Grant No. 51305210).

Open Access This article is distributed under the terms of the Creative Commons Attribution License which permits any use, distribution, and reproduction in any medium, provided the original author(s) and the source are credited.

\section{References}

1. Fan YS, Zhang LQ, Liu B (2004) Networked manufacturing and manufacturing network. Chin Mech Eng 15(19):1733-1738
2. Zhang ZL, Yuan WZ, Wang W (2006) Automotive service composition for manufacturing grid based on domain-specific ontology. $\mathrm{J}$ Comput Appl 26(1):57-60

3. Tan W, Fan YS (2005) Research on service matching and composition in networked manufacturing environment. Comput Integr Manuf Syst 11(10):1408-1413

4. Tao F, Hu YF, Zhao DM, Zhou ZD, Zhang HJ, Lei ZZ (2009) Study on manufacturing grid resource service QoS modeling and evaluation. Int J Adv Manuf Technol 41:1034-1042

5. Tao F, Hu YF, Zhao DM, Zhou ZD (2009) Application and modeling of resource service trust-QoS evaluation in manufacturing grid system. Int J Prod Res 47(6):1521-1550

6. Liu LL, Yu T, Shi ZB, Fang ML (2003) Self-organization manufacturing grid and its task scheduling algorithm. Comput Integr Manuf Syst 9(6):449-454

7. Shi ZB, Yu T, Liu LL (2004) Manufacturing grid and its resource configuration algorithm. Comput Eng 30(5):117-119

8. Yu T, Yuan LP, Li J, Xiong F, Fang ML (2005) A multi-agent based approach for manufacturing grid workflow. Proceedings of the fourth international conference on machine learning and cybernetics, Guangzhou, August 18-21: 199-204

9. Wen HY, Ren XL, Xu GH (2004) Study on searching algorithm of manufacturing grid. Chin Mech Eng 15(22):2014-2017

10. Deng H, Chen L, Wang CT, Deng QN (2006) A grid-based scheduling system of manufacturing resources for a virtual enterprise. Int $\mathrm{J}$ Adv Manuf Technol 28:137-141

11. Chen L, Deng H, Deng QN, Wu ZY (2004) A research of grid manufacturing and its application in custom artificial joint. Proceedings of International Conference of Computer Science 2004 (ICCS2004), April. Krakow, Poland, LNCS 3036:507-510

12. Zhang CS, Mo R, Shi SY, Chang ZY (2006) Research on manufacturing grid resource scheduling based on genetic algorithm. Chin Mech Eng 17(18):1916-1920

13. Lv BS, Shi SY, Mo R, Chang ZY, Yang HC (2006) Market equilibrium based resource optimal allocation for manufacturing grid. Comput Integr Manuf Syst 12(12):2011-2016

14. Fu JZ (2013) An efficient resource-searching method in manufacturing grid. Int J Adv Manuf Technol 66:401-405

15. Eldon H, William WG (2003) Global optimization using interval analysis. Marcel Dekker Inc, New York 